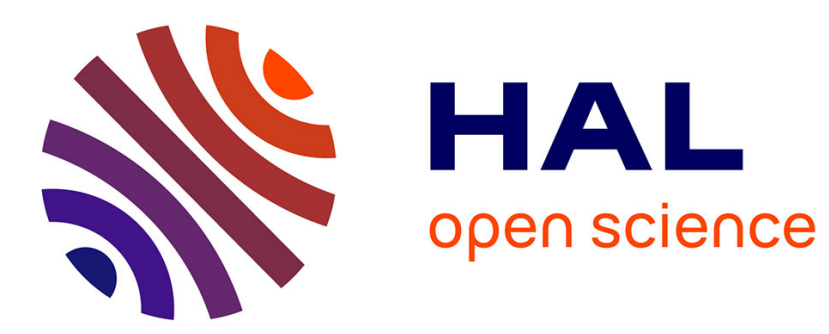

\title{
La construction identitaire des enseignants débutants et l'émancipation par rapport à la prescription
}

\author{
Jacques Méard, Philippe Zimmermann
}

\section{To cite this version:}

Jacques Méard, Philippe Zimmermann. La construction identitaire des enseignants débutants et l'émancipation par rapport à la prescription. Travail et apprentissages: revue de didactique professionnelle, 2013, 10.3917/ta.012.0065 . hal-03188364

\section{HAL Id: hal-03188364 \\ https://hal.science/hal-03188364}

Submitted on 1 Apr 2021

HAL is a multi-disciplinary open access archive for the deposit and dissemination of scientific research documents, whether they are published or not. The documents may come from teaching and research institutions in France or abroad, or from public or private research centers.
L'archive ouverte pluridisciplinaire HAL, est destinée au dépôt et à la diffusion de documents scientifiques de niveau recherche, publiés ou non, émanant des établissements d'enseignement et de recherche français ou étrangers, des laboratoires publics ou privés. 
La construction identitaire des enseignants débutants et l'émancipation par rapport à la prescription

Jacques Méard et Philippe Zimmermann

LAMHESS, EA 6309, Université de Nice Sophia Antipolis, France

\title{
Résumé
}

Cette recherche porte sur le façonnage de l'identité professionnelle (IP) de professeurs des écoles en formation initiale (PEFI) et vise à analyser comment s'effectue ce façonnage dans le cadre d'un cursus universitaire formant au métier d'enseignant. Le cadre théorique et la méthode prennent appui sur les postulats de la clinique de l'activité (Clot, 1999). Les résultats pointent l'importance de la renormalisation des prescriptions par les PEFI pour développer leur IP. La discussion porte sur leurs difficultés pour intégrer dans leur activité les prescriptions et se construire comme enseignants.

Mots-clés : développement - travail - enseignant stagiaire - apprentissage professionnel

About preservice teachers activity: identity process and freeing from prescriptions

\begin{abstract}
This article comes within a research program that focuses on the professional identity development of preservice teachers. It presents how this development takes place in a teacher training curriculum. The theorical and methodological framework is based on the clinical analysis of activity theory (Clot, 1999). Results show the importance for the preservice teachers to renormalize the prescriptions and to develop their professional identity. They position on difficulties for the preservice teachers to take into account prescriptions in their activity and to become teachers.
\end{abstract}

Key words : Activity Development - Work - Preservice Teachers - professional learning 


\section{Introduction}

Un des présupposés de la formation des adultes dans une perspective de professionnalisation, est sa visée de «construction identitaire ». Autrement dit, la formation à un métier implique non seulement une émancipation par rapport à une tutelle ou une autorité mais aussi par l'acquisition d'une compétence, au sens juridique du mot, l'établissement de sa propre « autorité ». L'homme ou la femme «de métier», à terme, se distingue par sa capacité à « s'autoriser », littéralement à « se faire son propre auteur » (Ardoino, 1977, p.74). Or, cette construction identitaire ne va pas de soi, car deux mécanismes apparemment contradictoires semblent la fonder : "l'identisation », c'est-à-dire la reconnaissance de la singularité du sujet, et «l'identification », c'est-à-dire la reconnaissance de sa similitude avec les autres (Dubar, 1996 ; Tap, 1988). Ainsi, l'apprenti deviendra «compagnon » à l'issue d'un itinéraire qui l'oblige à « faire comme », afin de maîtriser les règles de l'Art, puis progressivement à « faire à sa façon » pour marquer sa propre « autorité » (dont la signature sera son chef-d'œuvre).

On reconnaît là le paradoxe habituel de toute formation qui présente comme limpide le passage d'une étape d'obligation de faire, marquée par un rapport dissymétrique initial entre formateur et formé, vers un dénouement qui se caractérise par un pouvoir augmenté, une liberté acquise, une compétence transférée. Or, on oublie de dire que cette augmentation, cette acquisition, ce transfert, s'opèrent presque toujours dans la douleur. Ainsi, le formé ne se libère pas du rapport dissymétrique sans crise, il ne s'autonomise pas sans avoir mené bagarre, il ne reçoit pas une autorisation mais gagne plutôt son autorité.

Concernant l'identité professionnelle d'enseignants du premier degré en formation initiale, le processus qui recouvre l'ensemble des expériences vécues par l'étudiant puis l'enseignantstagiaire, à l'université et en stage, révèle ces mêmes paradoxes, et d'autres encore (Beijaard, Meijer, Verloop, 2004). Ainsi, dans le système de formation français actuel (voir la Circulaire du 25/02/2010), l'essentiel de ces expériences ont lieu à l'université. Cette situation place par exemple les fonctionnaires stagiaires de façon ponctuelle en responsabilité dans les classes, alors que leur formation n'est pas terminée et qu'ils n'ont pas été titularisés. Le plus intéressant dans ce cas précis tient dans le fait que les enseignants débutants sont d'anciens élèves. En un certain sens, leur manque d'expérience de travail, dans les classes, au cours de leur formation, est censé être compensé par leurs innombrables expériences d'élèves. L'enseignant débutant est supposé gagner son autorité professionnelle non pas tellement au contact de gens de métier qui l'accompagneraient mais à partir des souvenirs de ses expériences enfantines et adolescentes, c'est-à-dire du rapport dissymétrique dans lequel il a baigné (et réussi) quand il était élève. Sans parler de la confusion de perceptions, des 
renoncements et remises en cause provoquées par cette situation, l'inadéquation entre ses connaissances et sa compréhension de la classe (Flores \& Day, 2006) explique le choc fréquemment observé de l'entrée dans le métier (Veenman, 1984). Il ne suffit pas d'obtenir un diplôme pour «être enseignant». Il faut être est légitimé par les usagers et les membres du collectif de travail (Sutherland, Howard \& Markauskaite, 2010).

Autre spécificité, conjoncturelle cette fois : la construction identitaire de l'enseignant s'inscrit dans un contexte de remise en question du métier (voir par exemple le Rapport du Haut Conseil de l'Education: L'école primaire, Bilan des résultats de l'Ecole, 2007), de nonreconnaissance des compétences de l'enseignant par l'opinion publique (Martineau \& Presseau, 2007), d'accélération des changements des programmes (Goigoux, 2002), de réformes qui définissent de nouvelles tâches pour les enseignants (voir la Loi du 11 février 2005 ainsi que la Circulaire du 24 avril 2009). Ces évolutions multiplient les prescriptions, bouleversent le métier et l'on peut penser qu'elles ne sont pas sans effet sur la construction identitaire des enseignants.

Dans quelles circonstances la construction identitaire de l'enseignant débutant se réalise-telle ? Comment se déploie le processus d'émancipation qui la caractérise ? Pour répondre à ces questions, nous présentons ici les résultats d'une étude longitudinale menée auprès de six enseignants débutants.

\section{Cadre théorique}

Nous aborderons ces questions selon les postulats théoriques de la clinique de l'activité (Clot, 1999 ; 2008), elle-même inspirée de la psychologie culturaliste russe (Leontiev, 1976 ; Vygotski, 1985) et de l'ergonomie francophone (Daniellou, 2002). Ces postulats apportent un éclairage particulier sur l'émancipation qui est supposée sous-tendre le processus de construction identitaire. Ils reposent en effet sur le concept « d'activité » qui implique, chez le sujet, l'intériorisation de "signes externes » ayant une origine culturelle. Ainsi, Vygotski (1978) décrit un processus au cours duquel le sujet « apprend » puis « se développe » (c'est-àdire « greffe » ces signes, les intériorise pour devenir plus autonome). De là, on considère que les enseignants débutants, destinataires de ces «signes culturels » (les principes et techniques d'enseignement, les règles de l'art, ...) au cours des échanges, des moments de formation et en classe (l'interpsychique), les « greffent » pour en faire des instruments psychologiques, des ressources pour agir (l'intrapsychique). Ces signes sont destinés à devenir des outils de pensée et de jugement au cours de l'expérience professionnelle en classe. Dans cette optique, l'identité d'une personne ne repose pas sur des motifs d'agir singuliers. Elle est intelligible à 
l'intérieur d'une culture dans laquelle une personne agit et se développe (Daniels, 2007 ; Roth, 2007). Les actions sont dirigées vers soi et vers les autres à travers l'utilisation d'outils culturels. En retour, ces outils façonnent le fonctionnement individuel (Wertsch, 1991).

Pour comprendre le processus lui-même, il est nécessaire de recourir à l'organisation de l'activité telle que la décrit Leontiev (1976). D'après cet auteur, un sujet agit en fonction de buts («actions ») et ces actions sont indexées à la fois à des « mobiles vitaux» (ce qui pousse l'individu à agir) et à des « opérations » (ce que le sujet met en œuvre concrètement pour agir). L'identité professionnelle est donc comprise comme un développement de l'activité, c'est-à-dire un processus dynamique et situé de transformation des actions, mobiles et opérations. Dans ce modèle, les déplacements successifs des mobiles du sujet (j'agis pour garder le contrôle de la classe, puis pour motiver les élèves, puis pour les mettre au travail, etc.) et l'accroissement des opérations mises en œuvre (je vais présenter les consignes de cette façon, je parviens maintenant à agir de telle et telle façon avec les deux élèves perturbateurs de la classe) représentent les deux phases alternées du développement. Cette approche permet de concevoir la construction identitaire des enseignants débutants selon ce «processus biphasé » (Saujat, 2010) de développement «par les mobiles » et «par les opérations ».

Ce déplacement de mobiles et cet accroissement des opérations trouvent leur origine dans les conflits intrapsychiques que vit le sujet (Vygotski, 1978). Ceux-ci résultent d'une mise en concurrence des signes adressés par des interlocuteurs. Ils renvoient à un ensemble d' « actions concurrentes » possibles à réaliser (Bertone, Méard, Euzet, Ria \& Durand, 2003). Ces conflits intrapsychiques font envisager d'autres «préoccupations » (mobiles) et d'autres « manières de faire » (opérations). Ils alimentent momentanément des hésitations, des doutes, des « empêchements » (Clot, 1999) mais conduisent à un déplacement des mobiles et à la mise en œuvre de nouvelles opérations.

Enfin, la clinique de l'activité recourt au concept ergonomique de «prescriptions », conçues comme des "pressions diverses exercées sur l'activité d'autrui, de nature à en modifier l'orientation " (Daniellou, 2002, p. 11). Celui-ci permet de décrire un enseignant débutant cherchant des compromis entre les injonctions institutionnelles, les réalités de la classe et ses préoccupations personnelles. Pour se construire en tant que professeurs des écoles, les débutants doivent intégrer dans leur activité à la fois les prescriptions et les contraintes des collectifs professionnels, de la réalité du travail. Mais ils doivent surtout « renormaliser » ces prescriptions, c'est-à-dire opérer de multiples ajustements pour les adapter, les rendre vivables, les investir de sens, les «mettre à leur main ». 
En ce sens, l'émancipation dans la formation initiale des enseignants est pensée ici à la manière d'un processus de renormalisation des prescriptions qui conduit à une activité reconnue. Le but de cette communication est de mieux comprendre ce processus grâce à l'analyse longitudinale de l'activité de six enseignants débutants en conditions naturelles, notamment au travers des conflits intrapsychiques vécus au cours de leurs expériences en classe, face aux multiples prescriptions. En quoi ces situations leur permettent-elles ou non de construire leur identité ?

\section{Méthodologie}

\section{Participants}

Six enseignants débutants volontaires d'un Institut Universitaire de Formation des Maîtres ainsi que les élèves de leurs classes ont participé à cette recherche. Les enseignants étaient en dernière année de formation initiale, bénéficiaient d'une formation en alternance et $n$ 'avaient aucune expérience d'enseignement en responsabilité.

\section{Recueil des données}

Le recueil des données s'est effectué durant une année de formation. Quatre types de données ont été recueillis : a) Six enregistrements audio-vidéo (AV) avec chaque enseignant débutant, réalisés en contexte de formation et en classe lors des périodes de stage, b) des carnets de bord individuels, dans lesquels les enseignants notaient au moins deux fois par semaine les expériences signifiantes vécues, c) des curseurs placés sur des échelles 'enseignant' et 'étudiant' graduées de 0 (je me sens peu enseignant / étudiant) à 10 (je me sens pleinement enseignant / étudiant), inspirées des échelles d'auto-positionnement de Likert. Ces curseurs complétaient chaque expérience signifiante consignée dans le carnet de bord. La somme des deux curseurs ne devait pas nécessairement être égale à 10. Ces traces d'activité ont permis l'élaboration de graphiques (Schéma 1) et ont servi de supports à d) des entretiens d'auto confrontation (AC). Les graphiques étaient utilisés comme supports pour étayer ou introduire des controverses au cours des entretiens d'AC. Le développement de l'IP a été reconstruit à travers l'ensemble des données recueillies.

\section{Traitement des données}

Le traitement des données s'est déroulé en quatre étapes. Les données vidéo, carnets de bord et auto-positionnements des enseignants ont uniquement servi de supports aux entretiens 
d'AC. Dans un premier temps, ces entretiens ont été retranscrits verbatim. Puis des prescriptions ont été identifiées et formalisées à partir du repérage des mobiles vitaux, des opérations, des buts, et des éléments d'expérience de l'activité des débutants (Méard, Bertone \& Flavier, 2008). Les opérations correspondaient à ce qui était mis en oeuvre. Les buts représentaient l'objet de l'action (ex. : mettre les élèves au travail). Les éléments d'expérience étaient des éléments du contexte qui influaient sur la situation et étaient nécessaires à la compréhension de la situation dans sa complexité (ex. : " il fallait que ça arrive le jour de la visite »). La troisième étape a consisté à identifier les écarts entre ce qui est demandé et les mobiles vitaux à travers l'analyse des énoncés touchant à la façon de faire et aux raisons d'agir (auto-adressage de prescriptions). Enfin, trois chercheurs ont comparé et négocié leurs analyses dans un souci de validation des résultats obtenus. Les points de désaccord ont été discutés en vue d'obtenir un consensus. En cas de désaccord persistant, les éléments du corpus concernés ont été rejetés.

Résultats

Seule une partie des résultats de l'étude sera rapportée ici. Nous présenterons d'abord les effets de la formation sur la construction identitaire. Ensuite, nous pointerons le rôle des conflits intrapsychiques sur ce processus.

Les rapports dissymétriques comme entraves à la dynamique identitaire

Les résultats de l'étude montrent que le processus identitaire des enseignants débutants est marqué par l'évolution non linéaire de deux identités simultanées et concurrentes (enseignant et étudiant). Cette concurrence, loin d'être atténuée, est souvent suscitée par la formation ellemême. Ce résultat a été constaté auprès des six enseignants débutants, tout au long de l'année scolaire, tant dans les contextes de formation en centre qu'au cours des stages. Le cas de Sam, illustre ce résultat. Lors d'une visite de formateur au cours du stage $\left(9^{\text {ème }}\right.$ jour de stage en responsabilité effectué dans une classe de grande section - cours préparatoire), Sam est confrontée à la remarque d'un élève qui la surprend : « Mais maîtresse on n'y arrive pas ». L'entretien d'AC rend compte de l'activité contrariée de la débutante à ce moment (Extrait 1).

Extrait 1 - Extrait d'entretien d'AC (Sam, 27 novembre) :

$\mathrm{CH}$ : Qu'est-ce qu'il se passe à ce moment là [quand l'élève te dit «mais maîtresse on n'y arrive pas $\gg] ?(\ldots)$ 
SAM : Oui, bin, en fait, je me dis mince, qu'est ce que je vais faire, qu'est-ce que je vais... faire pour qu'ils y arrivent, et après je me dis... évidemment le jour de la visite, fallait que ça tombe. Et euh... après j'ai... et, là, on le voit bien, je suis en train de réfléchir à ce que je vais faire [opération] pour... pour qu'ils, qu'ils y arrivent et qu'ils se mettent au travail. Parce que finalement ils faisaient du bruit parce qu'ils y arrivaient pas. Et euh, c'est, au début, je m'en rendais pas compte, vu que je faisais pas attention à eux, et c'est vrai que, à ce moment-là, à la fin de la journée, j'ai dit à mon ATSEM qu'elle aurait pu venir me le dire tout de suite [opération]. Parce qu'alors j'aurais arrêté et puis j'aurais fait autre chose avec eux. Mais c'est vrai que là, je vais pas regarder [opération], je pensais qu'ils allaient y arriver parce qu'on avait déjà travaillé ça, je m'étais dit bon, c'est un peu difficile avec tous les morceaux et puis la qualité des images, mais après je me suis dit peut-être que, ça va quand même marcher, et finalement bon bin ça n'a pas marché, et là, quand il me dit « on n’y arrive pas », sur le coup je me... je... enfin je j'ai vraiment l'impression que je me décompose. Que je me dis oh mince, ça va, ça va plus, là. (...) Bin sur le coup je me dis, bon je continue, je fais comme si de rien n'était (rires), c'est c'est un peu bête mais bon... après je me dis ah, bin non, tu te fais visiter [mobile], là, faudrait peut-être réagir, et c'est là que je leur dis ah bon je vais venir vous aider [opération].

$\mathrm{CH}$ : Comment elle réagit, l'ATSEM, quand tu lui dis en fin de journée t'aurais pu venir me voir ...

SAM : (...) Elle me dit... oui, mais c'est pas mon rôle, euh, de savoir leur niveau. (...) Je lui ai bien fait comprendre, c'était pas à elle de réajuster, pas du tout, il fallait juste qu'elle, qu'elle me prévienne, qu'elle me dise que voilà ça marche pas, que..., que finalement ils restent pas pendant une demi heure à rien faire parce qu'ils y arrivent pas. (...) Ce serait sympa que des fois elle regarde et qu'elle me donne son opinion.

Au cours de cet extrait d'entretien, plusieurs éléments formulés par l'enseignante permettent de montrer que son activité est contrariée en raison d'un décalage entre ce qu'il s'est passé (les élèves ne parviennent pas à réaliser la tâche) et les attentes initiales : «je pensais qu'ils allaient y arriver parce qu'on avait déjà travaillé ça», "ça n'a pas marché ». Sam est confrontée au problème de l'ajustement du niveau de difficulté des situations à ses élèves et on se rend compte qu'elle ne maîtrise pas les opérations (techniques, outils professionnels) pour réaliser cet ajustement. La remarque de l'élève ne la perturberait pas davantage en temps normal. Mais dans le contexte d'une visite évaluative, cette remarque d'élève engendre un 
embarras typique «d'étudiant». Sam ne peut refuser l'aide aux élèves, parce qu'elle est en situation d'évaluation, mais elle ne peut l'apporter parce qu'elle est en déficit d'opération : "sur le coup je me dis, bon je continue, je fais comme si de rien n'était». Mais elle ne parvient pas à trouver une réponse efficace et réalise que la situation lui échappe.

L'embarras exprimé par Sam est renforcé par la présence de l'ATSEM, plus expérimentée qu'elle et qui connaît bien les élèves. Sam aurait aimé se référer à elle. Si l'ATSEM lui avait signalé que les élèves butaient sur leur travail, elle aurait évité de se retrouver dans une situation difficile: «ce serait sympa que des fois elle regarde et qu'elle me donne son opinion ».

Ce qui spécifie ce type de situation, c'est le déplacement de mobile : «agir pour faire mieux enseigner aux élèves » devenant «agir pour ne pas montrer ma faiblesse à l'évaluateur ». L'analyse de ce cas permet d'éclairer un des multiples glissements de l'identité « enseignante d'élèves » à celle d' « élève enseignante », tels que notre étude les a révélés dans l'activité des six enseignants participant à l'étude.

Le rôle des conflits intrapsychiques sur la construction identitaire

Une part très importante des données met en évidence que la concurrence entre les identités étudiante et enseignante se noue et se dénoue au travers de conflits intrapsychiques. Le cas de Tom, à la date du 15 décembre, illustre ce résultat. Au cours de son $13^{\text {ème }}$ jour de stage filé en petite section de maternelle, Tom constate que deux élèves discutent en turc lors d'un regroupement auquel participe également l'ATSEM de la classe (Agent Territorial Spécialisé des Ecoles Maternelles) (Extrait 2).

Extrait 2 - Extrait d'un entretien d'AC (Tom, 15 décembre)

TOM : Là, toutes les 2, oui, ça je sais qu'elles discutent en turc, j'ai même pas besoin de, je peux en être certain, ça c'est, c'est difficile, parce que ils parlent, donc en soi c'est quand même important, mais ils parlent la langue maternelle, donc nous, on nous dit il faut les laisser parler turc [action prescrite], parce que mieux ils parleront turc, mieux ils parleront français [mobile], mais en même temps quand tu fais une explication [opération] et qu'ils discutent en turc [élément d'expérience] c'est pas non plus acceptable, et même si ils discutent en français ou en, peu importe, mais...

CHERCHEUR $(\mathrm{CH})$ : Qui vous a dit qu'il faut les laisser parler... 
TOM : Oui, ça c'est en cours qu'on a, qu'on a demandé, dès le début de, de l'année, je crois c'est plusieurs professeurs qui nous ont conseillé de laisser parler les enfants dans leur langue maternelle [action prescrite], ce que, c'est c'est une question qui revient chez beaucoup de, de stagiaires, hein. Et c'est vrai que c'est, moi, je vois pour les, pour les ATSEM, parfois, c'est difficile à accepter, quand je leur dis ça. Parce que elles, elles disent aux enfants, mais vous parlez français. Et alors moi, je viens, tout frais sorti de l'IUFM je leur dis mais, oui mais il faut les laisser parler turc [action prescrite] parce que ... ah bin ouai mais oh la..., et puis alors il y a le discours un peu de celles qui ont de la bouteille qui revient, et des fois c'est, là-dessus elles sont pas d'accord avec moi. (...) Moi je pars du principe je débute. Donc je sais pas tout, et que ce qu'on m'a dit, a priori c'est vrai, mais en même temps je reste aussi méfiant, par rapport à ce qu'on me dit. Et finalement, je termine la discussion en disant bin moi c'est ce qu'on m'a dit à l'IUFM [action prescrite], a priori ça me semble pas faux, mais voilà, après, chacun ses idées. Je termine sur, sur une petite (rires), voilà

$\mathrm{CH}$ : T'essayes d'avoir un discours pas trop tranché, on va dire, ou t'essayes de pas trop t'opposer à elles?

TOM : Non, j'essaye quand même d'éviter [opération], et puis bon, entre collègues, je verrais pas l'intérêt non plus, si on travaille ensemble, autant qu'il y ait une bonne ambiance [mobile], mais voilà, c'est bien parce que ça empêche pas de dire ce qu'on pense, tout en laissant les autres penser ce qu'ils ont envie de penser [opération], et voilà, bon. Il faut pas non plus... (...) moi-même au départ, je, j'étais assez d'accord avec elles, parce que on est à l'école, dans les programmes, il faut apprendre la langue française [action prescrite], il faut une, et puis alors, début d'année, on arrive en cours ici, et puis alors non, il faut les laisser parler turc [action prescrite], c'est pas grave, ils se tromperont pas en français, ils seront d'autant, ils seront d'autant meilleurs en, en français et puis bon, moi, ça m'avait surpris, au début, hein. Alors pour l'instant, je, je sais pas, je suis un peu entre les deux. Mais quand ils parlent en turc, je, par exemple à l'accueil, dans des moments où ils peuvent parler entre eux, j'interviens pas du tout en leur disant vous parlez français [opération]. Ce que j'ai fait, les deux premiers lundis, je crois, au tout début, avant les cours en question. Alors je leur avais dit mais on est à l'école, on parle français, mais tu vois, un peu naturellement, et sans, sans vraiment réfléchir à ça.

$\mathrm{Au}$ cours de cet extrait, Tom commente une situation où plusieurs orientations de l'action s'offrent à lui : laisser les élèves s'exprimer en turc, leur langue maternelle, intervenir pour 
qu'ils écoutent, tolérer qu'ils parlent en français... Il se réfère à ce qu'il a appris à l'IUFM : en principe, il faudrait interdire les échanges («quand tu fais une explication et qu'ils discutent en turc c'est pas non plus acceptable »), mais on peut les tolérer dans certains cas («ils parlent, donc en soi c'est quand même important »). Là encore, les hésitations de Tom sont renforcées par la présence de l'ATSEM, plus expérimentée que lui et qui pense que les élèves doivent s'exprimer en français. Bien que Tom soit «d'accord» avec elle au départ, parce que les programmes préconisent l'apprentissage de la langue française, il avoue néanmoins être « surpris » par cet argument. Les données montrent que l'origine de l'indétermination de Tom trouve sa source dans l'hétérogénéité des prescriptions: les prescriptions officielles des programmes, celles de l'IUFM, considéré ici comme le prescripteur secondaire des programmes, mais aussi celles de l'ATSEM (qui n'est ni un pair, ni un membre prescripteur mais qui fait partie du collectif de travail). Pour dépasser ce conflit, l'enseignant effectue un compromis et laisse finalement les élèves s'exprimer en turc quand ils discutent entre eux. La prescription qu'il s'auto-adresse l'amène ainsi à modifier ses façons de faire. Le mobile relatif à une meilleure expression en français si le turc est bien parlé oriente ses actions et ses opérations. Il intervient également auprès de l'ATSEM pour lui signifier d'en faire de même, mais évite d'avoir un discours trop tranché et laisse «les autres penser ce qu'ils ont envie de penser». L'entente et l' «ambiance de classe» prennent ici le pas sur le suivi des prescriptions énoncées à l'IUFM. Mais les données mettent en évidence qu'au niveau des opérations, Tom procède à des ajustements (faire passer une explication dans le silence) avec les éléments de la situation pour réaliser des actions en lien avec ses mobiles (apprendre à parler français aux élèves).

\section{Discussion}

Les résultats présentés ici et illustrés par deux extraits de données, soulignent l'importance du collectif de travail dans le développement professionnel. Ce collectif fait intervenir une pluralité de prescripteurs qui alimentent des conflits intrapsychiques chez l'enseignant débutant. Les nombreux contextes dans lesquels se déploient les expériences de l'enseignant (l'IUFM, la classe du stage en responsabilité...) sont à l'origine du tiraillement entre ses propres valeurs et croyances, les normes prescrites par l'école républicaine et celles du collectif de travail. Ce « débat de normes » est essentiel pour apprendre le métier; le débutant doit s'approprier le travail prescrit, mais ne pas l'appliquer tel quel, s'en dégager pour construire sa propre «identisation » (Tap, 1988). C'est finalement en réalisant que le travail n'est pas le simple suivi de prescriptions que les enseignants débutants construisent leur 
identité professionnelle. En ce sens, les résultats de cette étude complètent plusieurs travaux relatifs aux effets des entretiens post-leçon (Bertone, Chaliès, Clarke \& Méard, 2006) ou des analyses de pratiques (Méard \& Bruno, 2009) sur le développement professionnel des enseignants débutants, travaux qui montrent que la formation participe à la construction identitaire dans certaines circonstances seulement, notamment lorsque le collectif de travail en stage offre des ressources et devient l'instance de renormalisation des prescriptions (Moussay, Flavier., Zimmermann \& Méard, 2011).

Mais on remarque que ces conflits issus de la situation tutorale (Moussay et al., 2011) participent à sa construction identitaire à la condition que les opérations à sa disposition permettent des compromis jugés acceptables par lui et par les membres du collectif de travail. Or, au cours des parcours de formation des six enseignants débutants, notre étude met en évidence que le processus est fréquemment interrompu, parfois sur des périodes de plusieurs semaines. Par exemple, lorsqu'une situation dissymétrique déplace les mobiles (les cas où une visite en stage indexe l'activité à des mobiles d'étudiant qui remplacent les mobiles habituels d'un enseignant), il y a entrave au façonnage identitaire. De même, lorsque le corpus des prescriptions qui sont adressées est trop dense, l'émancipation est empêchée car le conflit intrapsychique devient dilemme. En d'autres termes, il se résume à une alternative dans laquelle les deux termes opposés (par exemple, exclure du cours un élève perturbateur versus le garder en classe et l'ignorer) ne laissent pas la place à une issue, ne débouchent pas sur une troisième voie. L'un des principaux indicateurs de développement (sortir des conflits intrapsychiques par des compromis) peut disparaître au profit de solutions plus tranchées, copiées-collées de situations antérieures vécues dans son expérience d'élève. Ce résultat confirme les conclusions d'autres travaux (Méard et Bruno, 2008).

De même, si ces prescriptions ne portent que sur le quoi faire (et non le comment faire), l'enseignant débutant se sent démuni et ne peut mettre en œuvre d'opérations adaptées. Certains auteurs parlent ici de prescriptions discrétionnaires (Maggi, 2003), en ce sens que le travailleur est sommé de viser un but (par exemple, aménager des parcours personnalisés pour les élèves) sans avoir ni les savoir-faire, ni la maîtrise des instruments qui lui permettraient de l'atteindre. Ce déficit d'opérations rend les conflits intrapsychiques insolubles, et la «migration fonctionnelle de l'expérience» trop complexe (Vygotski, 1978). Ce manque d'efficience, marqué par des compromis opératoires introuvables, est alors fréquemment renforcé par le sentiment d'une non reconnaissance de sa professionnalité par les usagers (parents et même élèves) et par les membres du collectif de travail (ATSEM, autres enseignants). Ces différentes circonstances de formation sont certes observées dans d'autres 
formations professionnelles, par exemple dans les métiers de l'industrie ou des services. Dans le cas des enseignants, elles ont un effet puissant sur le processus identitaire de " l'apprenti » dans la mesure où elles réveillent chez lui de façon récurrente un conformisme d'étudiant. Son expérience de référence redevient son expérience d'ancien élève, le renvoyant en miroir à ce que vivent à leur tour ses élèves.

\section{Conclusion}

Concernant la thématique de l'émancipation en éducation, notamment dans les situations de formation professionnelle d'enseignants, la clinique de l'activité pose que la phase de « développement » proprement dite (affranchissement par rapport au signe externe que l'on a fait «sien» et dont on peut faire «autre chose») est précédée par une phase «d'apprentissage». Cette conception de la construction identitaire implique trois conséquences qui tranchent avec les perceptions communes en pédagogie et andragogie : d'abord, elle n'est possible que collectivement, l'émancipation individuelle en tant que telle n'étant souvent que le masque du conformisme (nous nous référons ici à la conception kantienne d'une autonomie définie comme «liberté sociale»). Le sujet se construit par le collectif. Deuxièmement, le développement de l'activité comme processus implique une phase critique (les conflits intra psychiques), ce qui éloigne des illusions d'une construction identitaire linéaire et harmonieuse et réhabilite les concepts de conflits, de controverses dans les problématiques de formation. Enfin, cette phase critique sous-entend l'installation d'une dépendance initiale, d'un ordre établi, d'un «déjà-là ». Et ce n'est pas le moindre des paradoxes que d'imaginer un rapport autoritaire, non plus seulement étape incontournable sur la voie de l'émancipation mais condition même de l'émancipation. On relève un paradoxe du même ordre dans plusieurs modèles de sciences humaines, telle la sociologie institutionnelle (pour qu'il y ait un «instituant», il faut un «institué » préalable) ou la psychanalyse freudienne (la dynamique oedipienne est finalement possible à la condition d'un rapport autoritaire initial). Mais si ce rapport dissymétrique est véritablement un préalable à la construction identitaire, il comporte toujours un risque d'aliénation, comme le souligne Vygotski (1978).

\section{Références}

Ardoino, J. (1977). Education et politique. Propos sur l'éducation II. Paris : Gauthier-Villars. Beijaard, D., Meijer P. C., Verloop, N. (2004). Reconsidering research on teachers' professional identity. Teaching and Teacher Education, 20, 107-128. 
Bertone, S., Chaliès, S. Clarke, A \& Méard, J. (2006). The dynamics of interaction during post-lesson conferences and the development of professional activity: Study of a preservice physical education teacher and her co-operating teacher, Asia-Pacific Journal Of Teacher Education (AUS), 34, (2), 245-264

Bertone, S., Méard, J., Euzet, J.- P., Ria, L. \& Durand, M. (2003). Intrapsychic conflict experienced by a preservice teacher during classroom interactions : a case study in physical education. Teaching and Teacher Education, 19, 113-125.

Clot, Y. (1999). La fonction psychologique du travail. Paris : PUF.

Clot, Y. (2008). Travail et pouvoir d'agir. Paris : PUF.

Daniellou, F. (2002). Le travail des prescriptions, In Actes du 37ième Congrès de la SELF " Nouvelles formes de travail, nouvelles formes d'analyse », Aix en Provence, 9-16.

Daniels, H. (2007). Discourse and identity in Cultural-Historical Activity Theory: A response. International Journal of Educational Research, 46, 94-99.

Dubar, C. (1996). La socialisation, construction des identités sociales et professionnelles. Paris: Armand Colin.

Flores, M. A., Day, Ch. (2006). Contexts which shape and reshape new teacher's identities: A multi-perspective study. Teaching and Teacher Education, 22, 219-232.

Goigoux, R. (2002). L'évolution de la prescription adressée aux instituteurs : l'exemple de

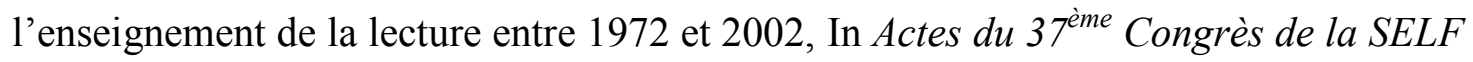
" Prescription et travail », Aix-en-Provence, 78-85.

Leontiev, A. (1976). Le développement du psychisme. Paris : Editions sociales.

Maggi, B. (2003). De l'agir organisationnel. Un point de vue sur le travail, le bien-être, l'apprentissage. Toulouse : Octarès.

Martineau, S, Presseau, A. (2007). Se «mettre en mots » ou se bricoler une histoire pour qu'elle prenne sens : le discours identitaire d'enseignants du secondaire. Dans Identités professionnelles d'acteurs de l'enseignement. Regards croisés, Ch. Gohier (sous la direction de), Québec, Presses de l'Université du Québec, 67-88.

Méard, J., Bertone, S., Flavier E. (2008). How fourth grade-students internalize rules during teacher-student(s) transactions. British Journal of Educational Psychology, 78, 395410.

Méard, J., Bruno, F. (2008). Le travail multi-prescrit des enseignants en milieu scolaire : analyse de l'activité d'une professeure d'école stagiaire. Travail et formation en éducation, 2. http://tfe.revues.org/index 718.html. 
Méard, J., Bruno, F. (2009). Les règles du métier dans la formation des enseignants débutants. Toulouse : Octarès.

Moussay, S. Flavier, E., Zimmermann, P. \& Méard, J. (2011). Preservice teacher's greater power to act in the classroom: analysis of the circumstances for professional development through sense and efficiency. European Journal of Teacher Education, 34. $4,385-400$.

Roth, W.-M. (2007). The ethico-moral nature of identity: Prolegomena to the development of third-generation Cultural-Historical Activity Theory. International Journal of Educational Research, 46, 83-93.

Saujat, F. (2010). Travail, formation et développement des professionnels de l'éducation : voies de recherche en sciences de l'éducation. HDR, Université Aix Marseille, non publiée.

Sutherland L., Howard S., Markauskaite L. (2010). Professional identity creation: Examining the development of beginning preservice teachers' understanding of their work as teachers. Teaching and Teacher Education, 26, 455-465.

Tap, P. (1988). La société Pygmalion ? Intégration sociale et réalisation de la personne. Paris, Dunod.

Veenman, S. (1984). Perceived Problems of Beginning Teachers. Review of Educational Research, 54(2), 143 -178.

Vygotski, L. S. (1978). Mind in society. The development of higher psychological processes. Cambridge : Havard University Press.

Vygotski, L. S. (1985). Pensée et langage. Paris : Editions sociales.

Wertsch, J. V. (1991). Voices of the mind: A socio-cultural approach to mediated action. Cambridge, MA: Harvard University Press.

\section{*REF à Zimmermann CDE}

